\title{
Hepatitis C Treatment: Shorter and Better?
}

\author{
Evaldo Stanislau Affonso de Araújo ${ }^{1,2}$, Cláudia Courtouké ${ }^{1}$ and Antonio Alci Barone ${ }^{1,2}$ \\ ${ }^{1}$ Outpatient Hepatitis Clinic, Department of Infectious and Parasitic Diseases, Hospital das Clínicas, University of São Paulo, School of \\ Medicine; ${ }^{2}$ Laboratory for Medical Investigation 47, University of São Paulo, School of Medicine; São Paulo, SP, Brazil
}

\begin{abstract}
Herein, we present a synthesis of two publications that evaluate an abbreviated therapeutic approach to treating chronic hepatitis $\mathrm{C}$ virus (HCV) infection. Based on those publications, we discuss the use of the early virologic response (EVR) as a tool for the optimized management of patients under treatment, as well as reviewing concepts of HCV viral kinetics. The fourth-week EVR, characterized by HCV RNA dropping to undetectable levels, allows individuals infected with HCV genotype 1 and presenting low baseline viral loads to be treated with the combination of pegylated interferon and ribavirin for 24 weeks, whereas individuals infected with HCV genotypes 2 or 3 can be treated for only 12 weeks. Therefore, by adopting abbreviated treatment regimens optimized through early prediction of sustained viral response, it is possible to increase the number of patients treated without incurring the excess costs related to high rates of treatment failure and management of adverse outcomes, as well as avoiding the risks of unnecessarily exposing patients to drugs that have the potential to be highly toxic.
\end{abstract}

Key-Words: HCV, interferon, viral kinetics.

Among the infectious diseases that are currently most common, infection with hepatitis C virus (HCV) presents one of the greatest challenges. According to the World Health Organization, approximately 170 million individuals worldwide are infected with HCV [1]. In Brazil, the number of HCV-positive individuals has been estimated at 3 to 4 million, 140,000 residing in the city of São Paulo [2]. Chronic hepatitis $C$ has potentially devastating consequences for public health, from the financial standpoint (due to the increased costs associated with antiviral treatment) and from the medical standpoint (due to complications associated with chronic end-stage liver disease).

The search for an equilibrium between the need of better diagnose, or rather to increase patient access to diagnostic services, as well as to offer effective treatment to a greater number of HCV-positive patients is a complex task. Approaches that better combine diagnosis and treatment, optimizing the currently available therapeutic arsenal (interferon and ribavirin), would undoubtedly be of great value. Within this context, the study of HCV kinetics during treatment raises issues that merit thorough analysis, which will certainly result in new strategies for managing chronic hepatitis $\mathrm{C}$ patients, matching diagnostic services access to optimized treatment regimens.

\section{Viral Kinetics}

Once chronic HCV infection has been established, a state of dynamic equilibrium is developed in which the rate of new viral particle production is equal to that of their destruction, and variations in viral loads quantified at different time points are less than $\log _{10} 0.5$ [3]. The virion half-life is 2.7 hours,

Received on 24 October 2006; revised 15 December 2006.

Address for correspondence: Dr. Evaldo Stanislau Affonso de Araújo. Instituto de Medicina Tropical, LIM 47. Av. Dr. Enéas de Carvalho Aguiar $n^{\circ} 500$ sala 12. Cerqueira César, São Paulo, SP. Zip code: 05403000. Phone/Fax: (5511)30851601. E-mail: evaldostanislau@uol.com.br.

The Brazilian Journal of Infectious Diseases 2007;11(1):118-124. (C) 2007 by The Brazilian Journal of Infectious Diseases and Contexto Publishing. All rights reserved. meaning that all circulating virions are eliminated and substituted within a three-hour interval, which translates to the production and elimination of $10^{12}$ viral particles/day [4]. In this state of equilibrium, the HCV is distributed to various real or virtual compartments: blood, liver, extrahepatic sites and the viral elimination compartment (Figure 1). Upon the introduction of the antiviral treatment, disequilibrium occurs in the system, resulting in a drastic reduction of the plasma component (lowering viral load). This initial stage of the treatment is characterized by the blocking effect that interferon has on the release of new particles from the infected hepatocytes and by the elimination of circulating viral particles. These phenomena occur between 24 and 48 hours after the introduction of interferon and characterize phase 1 of the viral decay (Figure 2). Depending on the magnitude and speed of phase 1 , this might represent, for many future patients, the acquisition of a sustained virologic response (SVR), as highlighted here and discussed below. After the elimination of infected cells (hepatocytes), perpetuators of the infection, phase 2 of the viral decay begins. This is a more gradual phase, in which ribavirin apparently plays a crucial role. Finally, in some cases, there is a plateau between phases 1 and 2. At this point, the viral decay is interrupted for a matter of hours or days, after which it enters phase 2 and continues its gradual decay [5]. Very recently, Tang et al. [6] proposed an explanation that allows a better understanding of how this occurs. According to those authors the very rapid activation of Interferon Stimulated Genes (ISG) after interferon alpha administration [7] and interferon attachment to interferon alpha receptors (IFNAR) - which in turn initiate a strong innate immune response [8] - it is possible to explain the effect of interferon alpha as a potent immunstimulant of an innate response with the very first few hours and of an adaptative response after the first four weeks. The magnitude of ISGinduced innate response allows a reduction on HCV viral load wich, if great enough, causes the subsequent effective CD4 and CD8 responses in a very distinct way between rapid responders (those presenting negativity for HCV RNA by 
Figure 1. Illustration of the three main compartments in HCV infection: compartment 1 is characterized by infected hepatocytes and extrahepatic sites; compartment 2 represents the circulating viral pool in the blood; and compartment 3 is the virtual compartment, in which the destruction of viral particles and infected cells occurs. The constant renewal of healthy and infected hepatocytes, as well as the infection of healthy hepatocytes and superinfection of infected hepatocytes, is also shown.

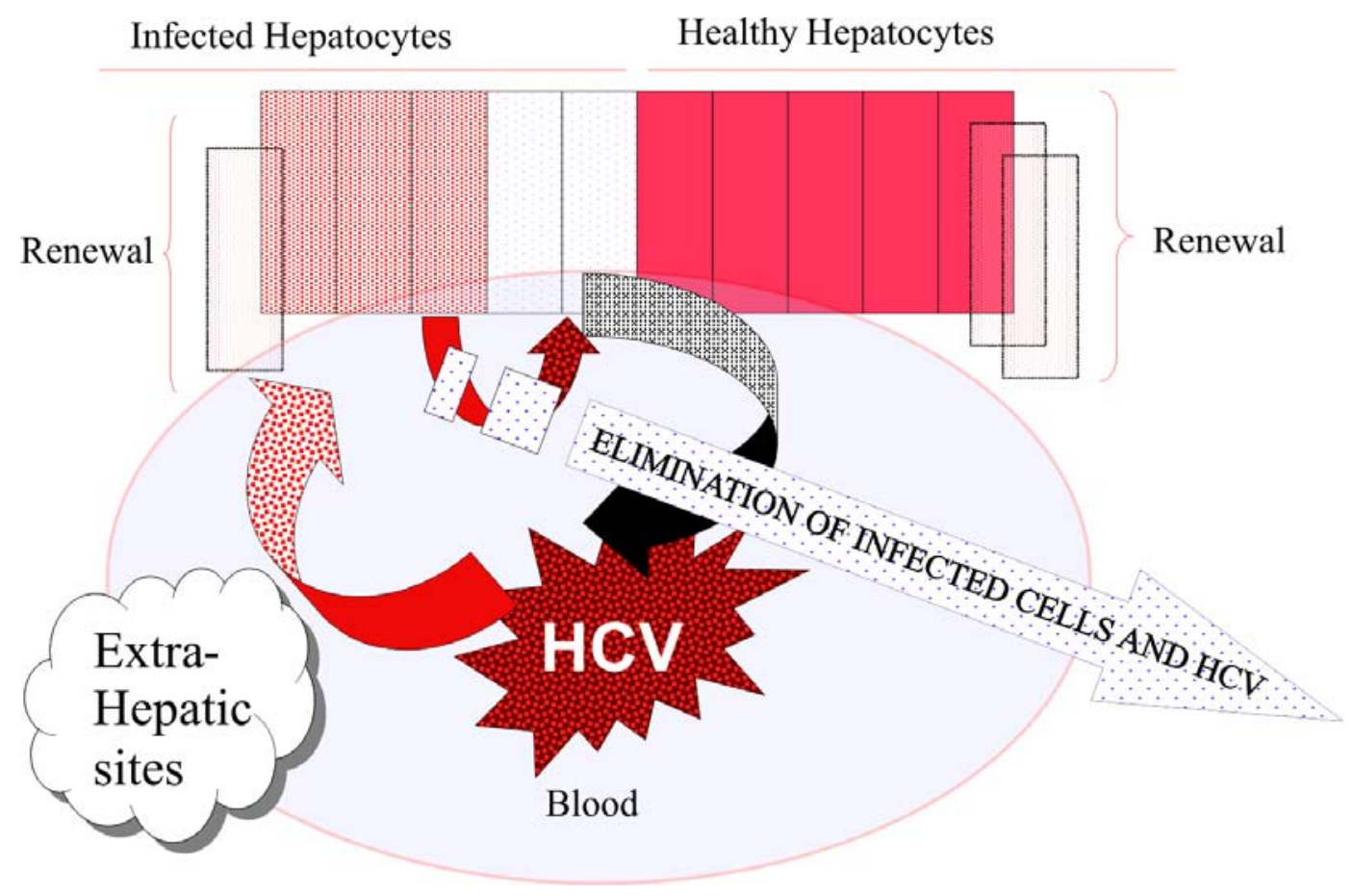

Figure 2. Viral kinetic curve: phase 1 and phase 2.

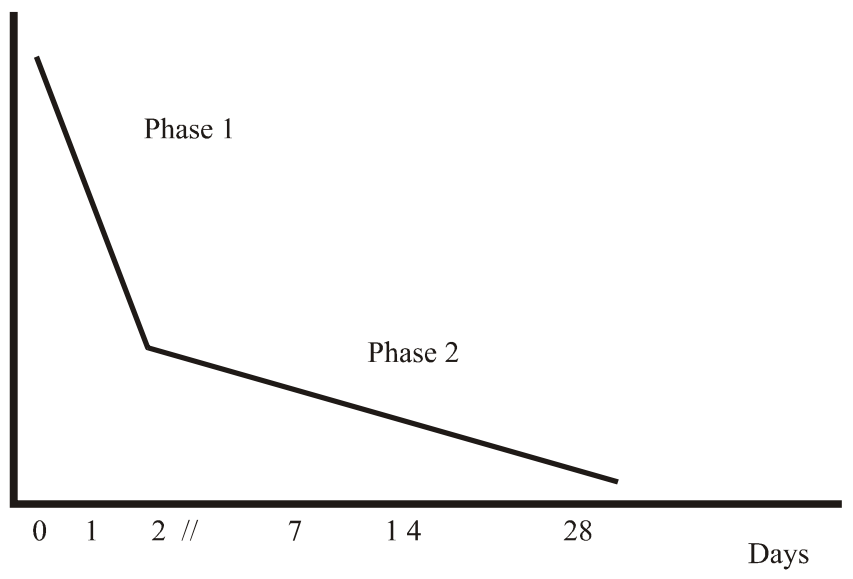

Figure 3. The steady steate is disrupted by the initiation of interferon-alpha treatment leading to progressive immune activation from innate to adaptive response through ISG stimulation.

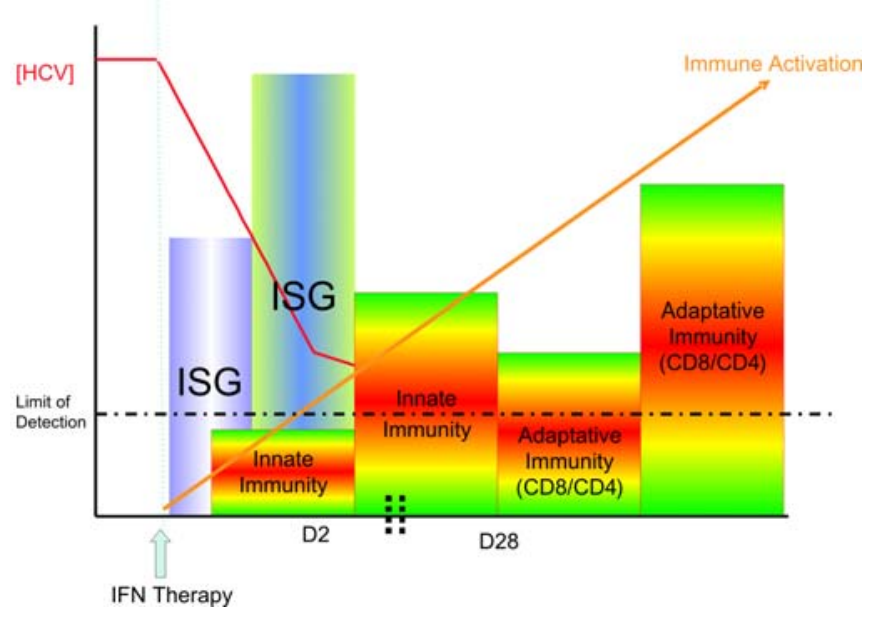


week 4 of treatment) with a strong activation, and slow responders, with a weak answer [6] (Figure 3). The reason why those distinct patterns exists as a result of various factors including those related to the host (genetic characteristcs, gender, race, weight, liver fibrosis, steatosis, etc.), to viral proteins and to pharmacology of the interferon-alpha (type of pegylation, receptor attachment, plasma level, etc.).

Starting from these basic concepts, and using the equation proposed by Neumann et al. [9], it is possible to calculate, mathematically, the kinetic parameters of HCV, two of which (interferon efficiency and cell death rate) are fundamental to phases 1 and 2. In practical terms, studies of viral kinetics are characterized by the frequent collection of blood samples and viral quantification at various time points during the initial phases of the treatment. This technique allows the recognition of viral decay patterns and the calculation of kinetic parameters. Based on those data, the response to the treatment can be predicted. Such studies are laborious and expensive, requiring their own logistics (that typically results in small study samples, making it difficult to assign statistical significance to the results obtained). However, these and other limitations do not decrease the relevance of such data or affect their concordance with those of previous studies, nor do they minimize the impact of the results. Moreover, kinetic studies with only viral load measurements - provides the knowledge on virus-host-drugs interactions. A better kinetic pattern (a rapid, pronounced decline in viral load) could be interpreted as a good interaction whereas a worse pattern (a slow decline) could require other therapeutic approaches, such as the prolongation of treatment.

\section{Viral Response Types}

When planning individualized antiviral treatment regimens, one must keep in mind the intended objective, whether viral or histological. Furthermore, when the fact that the hepatitis $\mathrm{C}$ treatment is limited in efficiency, as well as the costs and adverse effects of that treatment, is taken into consideration, the need for tools that allow early prediction of the response to the viral treatment becomes obvious. Such tools can not only diminish the understandable anxiety of the patients but can also be used to evaluate the appropriateness, cost effectiveness and maintenance of treatment regimens that can be difficult to manage (due to drug toxicity, drug interactions, costs and other limiting factors).

Taking into consideration virologic objectives, the possible responses are early virologic response (EVR), which will be discussed separately, the end-of-treatment response (ETR), the nonsustained (or recurrent) virologic response (NSVR) and the sustained virologic response (SVR). The SVR is the main objective, although its significance needs to be made clear. It is defined as a viral RNA level, as determined by standard methods, below the level of detection at six months after the end of the treatment, rather than as a "cure" in the sense of complete eradication of HCV. This is an important point that we have been making for some time based on the report made by Pham et al. [10], in which the authors found replicating viral particles up to five years after the SVR had been achieved. This finding has recently been corroborated in similar studies and in a Hepatology editorial entitled " $\mathrm{HCV}$ persistence: cure is still a four-letter word" [11], in which, despite of the lack of a fully-formed notion of the meaning of such persistence, the authors highlight the obvious impact that patients representing perpetual sources of HCV dissemination would have on public health, as well as the potential increased incidence of hepatocarcinoma.

In predicting the response prior to the initiation of the treatment, demographic, clinical, viral and even behavioral factors have to be taken in consideration. For example, the response is more predictable in nonobese, white female patients under the age of 40 who are able to adhere well to the treatment than in older, obese, black males who are unable to adhere fully to the treatment. The absence of coinfection with HIV is another favorable factor. Patients presenting lower hepatic fibrosis indices are also considered to have better prognoses than do those with more advanced fibrosis (exceeding the septal) [12]. Finally, individuals infected with HCV genotypes 2 or 3 are considered better candidates for success than those infected with genotypes 1 and 4 . Similarly, individuals presenting lower baseline viral loads $(<800,000$ $850,000 \mathrm{IU} / \mathrm{mL}$ ) are better candidates for success than those with higher baseline viral loads. This last factor (baseline viral load), is associated with kinetics, as we have been highlighting.

The EVR is considered not only a type of response in itself but also a predictor of SVR. It is possible that the EVR will surpass all of the other predictors mentioned above as a predictor of SVR, in particular when a individualized response model, in which the acquisition of a set pattern of viremia reduction at certain time points during the treatment would correlate in a positive or negative manner with the presence of SVR, is adopted. The EVR can be classified according to the time point at which it was determined. We have routinely defined the EVR pattern as the undetectability of HCV RNA, or a $\geq 2 \log$ decrease in the same, by week 12 of pegylated interferon treatment. The absence of this response has a negative predictive value (NPV) of $97 \%$. When this value is not attained, it is recommended, for patients monoinfected with $\mathrm{HCV}$ as well as for those coinfected with HIV and HCV, that treatment be discontinued [13,14]. However, the positive predictive value (PPV) at week 12 is not ideal - only $65 \%$ for those monoinfected [13]. In view of this, new time points are sought to predict the SVR in a more consistent manner. Therefore, the $\geq 2 \log$ decrease in, or undetectability of, HCV RNA can also be used as a criterion for week 4 of treatment. This would also have the obvious advantage of minimizing unnecessary drug exposure, as well as reducing the consequent adverse effects and high costs. However, as an important consideration for the clinical practice, it is of note that undetectability at week 4 is significantly more associated with SVR than is the simple $\geq 2 \log$ decrease $-87 \%$ vs. $52 \%$ for pegylated interferon and ribavirin, respectively, in the study conducted by Ferenci et al. [13]. 
Therefore, the introduction of qualitative investigation at week 4 , together with quantification at week 12 , can be a measurement to be taken into consideration in clinical therapeutic protocols. Finally, the relevance of adherence must be stressed. Drug exposure, especially in the first weeks of treatment, is associated with SVR. Therefore, rapid and vigorous reduction of the viral load in the first four weeks of pegylated interferon and ribavirin treatment is essential.

We recently showed that, in patients coinfected with HIV and HCV and treated with an optimal dose of pegylated interferon alpha 2a and ribavirin, an NPV for SVR of $100 \%$ can be achieved within 24 hours when a 1 log reduction is not observed. Alternately, if we use a 2-log decrease as the criterion, an NPV of $92.31 \%$ can be achieved by day 8 of the treatment and of $100 \%$ from week 4 onward [15]. So we can conclud that EVR is the best predictor of SVR, should be used earlier and enhances the cost-effectiveness of therapy representing an excellent tool for increasing access to treatment. However, patients with advanced hepatic lesions and those that present a delayed viral response can benefit from the results of ongoing studies that evaluate interferon maintenance or prolonged treatment, thereby reducing, respectively, the histological progression and the recurrence rate.

\section{The EVR in Practical Terms: Two Good Examples of Its Application}

Two recent publications $[16,17]$ presented two studies that were designed based on information originating from studies of viral kinetics demonstrating the relevance of the first phases of treatment in obtaining SVR, and of the potential for good predictability from a rapid and vigorous virologic response detected by week 4 of treatment. Those studies clearly showed the benefits of an optimized approach for patients infected with genotypes 1,2 or 3, all of them treated for shorter periods than those recommended in the current treatment protocols, exemplifying, in practice, good strategies to reconcile the collectively desired objectives to increase access to diagnostic services and treatment.

Mangia et al. [16] conducted a clinical randomized study involving chronic hepatitis $\mathrm{C}$ patients infected with genotypes 2 or 3, comparing the standard 24-week regimen of pegylated interferon alpha $2 \mathrm{~b}$ and ribavirin versus variable duration treatment based on EVR by week 4. In patients presenting EVR by week 4, treatment was discontinued after 12 weeks, whereas those not presenting EVR by week 4 were allowed to complete the standard 24-week treatment regimen.

That was an open study, performed in 12 centers in Italy between June 2002 and January 2004, receiving no financial support from the pharmaceutical industry and approved by the local ethics committee in agreement with good clinical research practices. The inclusion criteria were as follows: being monoinfected witg genotype 2 or 3; treatment-naïve; being 18 to 70 years of age; and having no other liver diseases, blood cytopenias or other conditions that would preclude IFN therapy. The study randomized 283 patients, 70 to standard therapy and 213 to variable duration, of whom 133 were HCV RNA negative at week 4 and were treated for 12 weeks. Baseline HCV load were $>600,000 \mathrm{IU} / \mathrm{mL}$ for $16 \%$, $95 \%$ were caucasian and the mean age was 42,2 years. The patients were randomly assigned, at a ratio of $1: 3$, to the variableduration group ( \pm 210 subject) or to the standard-duration (control) group ( \pm 70 subjects). All subjects received pegylated interferon alpha $2 \mathrm{~b}$ at a dose of $1 \mathrm{mg} / \mathrm{kg} /$ week and daily doses of ribavirin at $1 \mathrm{~g}$ for those with a body weight $<75 \mathrm{~kg}$ or $1.2 \mathrm{~g}$ for those with a body weight $\geq 75 \mathrm{~kg}$. In the control group, 66 patients completed the treatment, compared with 208 in the variable duration group: 132 from the short-term treatment subgroup (those presenting EVR by week 4) and 76 from the long-term treatment subgroup (those presenting no EVR by week 4), which completed the 24 weeks of treatment. The patients who dropped out of the study were classified as not presenting a virologic response. Blood samples were collected at weeks 4, 12 and 24, as well as at 24 weeks after the end of treatment, and were subsequently submitted to qualitatively analysis (Amplicor ${ }^{\circledR} \mathrm{HCV}$ test, version 2.0; Roche, Mannheim, Germany) and quantitative analysis in relation to baseline values (COBAS MONITOR ${ }^{\circledR}$ test, version 2.0; Roche).

The analysis of the results showed similarity in relation to baseline data. The percentages of patients presenting EVR were comparable between the standard-duration and variableduration groups (64\% and $62 \%$, respectively). In the standardduration group, $76 \%$ obtained SVR, compared with $77 \%$ in the variable-duration group, indicating that there was no inferiority in the short-term treatment subgroup. More importantly, within the variable-duration group, SVR was achieved by $91 \%$ of the long-term treatment subgroup patients and by $85 \%$ of the short-term treatment subgroup patients. The $6 \%$ difference was within the estimated confidence interval and therefore met the efficiency criterion for the proposed treatment. Among the standard-duration group patients not presenting EVR, $48 \%$ achieved SVR, whereas, in the variable-duration group patients not presenting EVR and therefore treated for 24 weeks, 51\% achieved SVR, meaning that the absence of EVR was correlated with a lower percentage of SVR. Recurrence was similar between the short-term treatment subgroup and the standard-duration-presenting-EVR subgroup $(\mathrm{p}=0.19)$. Patients in the short-term treatment subgroup who presented recurrence were given the option of being retreated for the full 24 weeks. Of the 13 in this situation, 10 accepted and 9 achieved SVR. None of the baseline characteristics - age, gender, body mass index, alanine aminotransferase (ALT), viral load, genotype (2 or 3), steatosis or fibrosis - were predictive of recurrence in the short-term treatment subgroup. Adverse effects were relatively infrequent, even less so in the shortterm treatment subgroup, which also had fewer exclusions related to adverse effects than did the long-term treatment subgroup ( 1 vs. $8, \mathrm{p}=0.045)$. In the univariate analysis of baseline factors previously described, together with that of EVR, revealed that baseline viremia $<800,000 \mathrm{IU} / \mathrm{mL}$ was 
associated with SVR ( $\mathrm{p}=0.049$ ), whereas no association that would be predictive of EVR was found in the multivariate analysis. It was therefore concluded that patients infected with genotypes 2 or 3 and presenting EVR by week 4 can be treated for 12 weeks with the same success rate and lower exposure to the risk of adverse effects, treatment-related inconvenience and, obviously, at a lower cost. Although there was greater recurrence in the short-term treatment subgroup than in the long-term treatment subgroup, an additional 24 weeks of treatment resulted in a 90\% rate of SVR among those patients. Finally, it is noteworthy that the pegylated interferon alpha $2 \mathrm{~b}$ dosage used was $1 \mathrm{mg}$ rather than $1.5 \mu \mathrm{g}$, representing additional savings and producing results similar to those obtained in the study that used the higher $(1.5 \mu \mathrm{g})$ dosage [18].

In a similar study, albeit involving individuals infected with HCV genotype 1 (which is more prevalent and harder to treat), Zeuzem et al. [17] showed good results using a 24-week course of treatment in patients with low baseline viral loads and presenting EVR. That was an open study conducted from 2001 to 2004 in 43 European centers. The inclusion criteria were being between 18 and 70 years of age, having chronic compensated hepatitis $\mathrm{C}$, being HCV treatment naïve, presenting a viral load of $<600,000 \mathrm{IU} / \mathrm{mL}$, recent liver biopsy showing chronic hepatitis, elevated ALT and no hematologic contraindications. Standard exclusion, safety and coinfection criteria were adopted. The patients received pegylated interferon alpha $2 \mathrm{~b}$ at a dose of $1.5 \mu \mathrm{g} / \mathrm{kg} /$ week and ribavirin at $800-1,400 \mathrm{mg} / \mathrm{day}$, according to body mass $(10.6 \mu \mathrm{g} / \mathrm{kg} /$ day). Treatment was given for 24 weeks. The study followed ethical and good clinical practice criteria. The study enrolled 237 patients of whom 214 completed therapy and follow up.

Analysis of the results based on the intention to treat showed that $80 \%$ of the patients achieved ETR, and 50\% (117 of the 235 patients) achieved SVR. The percentage of SVR obtained (69\%) was lower than what would have been expected had the patients been treated for 48 weeks. Therefore, a 24week treatment regimen was not sufficient to achieve SVR. Normal levels of ALT were seen in 57\% of the patients (134 of 235), and 47\% (110 of 235) presented normal ALT levels and undetectable RNA. In the univariate analysis, SVR was found to be associated with age, low baseline viremia and treatment for at least 16 weeks. However, in the multivariate analysis, only baseline viremia $\leq 600,000 \mathrm{IU} / \mathrm{mL}$ and treatment for at least 16 weeks were associated with SVR $(\mathrm{p}<0.0001$ and $\mathrm{p}=0.0135$, respectively). An important predictive factor was the lack of detectable virus at 4 weeks after the treatment had started. Of the patients presenting undetectable levels of HCV RNA by week 4 of treatment, $89 \%$ achieved SVR, compared with $25 \%$ and $17 \%$, respectively, for those presenting undetectable levels only by week 12 or 24 . Of the patients presenting undetectable levels of viral RNA by week 4 and at week 24, 92\% achieved SVR. There was also an association between baseline viral load $\leq 250,000 \mathrm{IU} / \mathrm{mL}$ and SVR ( $67 \%$ vs . $25 \%$ for those presenting viral loads $>250,000 \mathrm{IU} / \mathrm{mL}$ ), as well as an association between SVR and viremia that was $\leq 250,000$ $\mathrm{IU} / \mathrm{mL}$ at baseline and undetectable at week 4 (68\% vs. 16\% for those presenting viral loads $>250,000 \mathrm{IU} / \mathrm{mL}$ ).

The incidence of adverse effects was similar to that seen for historical controls. However, adverse events that resulted in the discontinuation of treatment or dose modification were lower than in historical controls treated for 48 weeks ( $3 \%$ vs. $14 \%$ and $26 \%$ vs. $49 \%$, respectively), even when only the first 24 weeks of the treatment were taken into consideration (11 versus $41 \%)$.

Despite of the fact that the short-term treatment was insufficient to achieve SVR in those infected with genotype 1 and presenting low viremia, it can be considered when a baseline viremia cut-off level of 250,000 IU/mL, rather than $600,000 \mathrm{IU} / \mathrm{mL}$, is used. The most consistent datum, however, is the predictability of presenting EVR by week 4, characterized by undetectable levels of viral RNA, which, when accompanied by low viremia, achieves an SVR rate of $90 \%$ (when compared to the $85 \%$ rate achieved in the historical controls treated for 48 weeks). Therefore, the subgroup with low viremia and presenting EVR by week 4 can be treated for 24 weeks, without jeopardizing the possibility of achieving SVR, with lower toxicity and, evidently, at a lower cost. Finally, it is of note that presenting EVR by week 4 can be considered the best predictor of SVR in clinical practice.

\section{Conclusions}

To the question implied in the title of this manuscript ("Can we expand and improve hepatitis $C$ treatment with shorter therapies?”), we answer, “Yes.” We live in a new era of hepatitis $\mathrm{C}$ treatment, the era of rationality, which has superseded the "Pegylation Era", in which we treated individuals who did not require treatment as well as those who did. Currently, physicians have more experience in the management of patients, have a more critical view of treatment, are attentive to disease progression (maybe benign for the majority) and know how to prioritize patients on the "list" of those requiring access to treatment (undoubtedly patients with more fibrosis and coinfected with HIV). In addition, sensitive and reproducible quantification methodologies are now widely available. Therefore, the concept of an individualized approach, avoiding overtreatment, could be easily understood and adopted. In addition, the studies reviewed herein showed that a 12-week treatment strategy is clearly justifiable for individuals infected with genotypes 2 or 3, with no current access to pegylated interferon. Such a strategy represents improved access to treatment that, if not more efficacious, is certainly more accommodating, with less exposure to adverse effects, and could be more cost-effective. Therefore, for individuals infected with genotypes 2 or 3, this strategy could expand access to treatment and improves the quality of that treatment. For the more prevalent genotype (genotype 1), NPV reaches nearly $100 \%$ when the criterion used is the presence of EVR by week 12 . However, 15 to $20 \%$ of those patients [19] present low viremia and could be treated for 24 weeks only. In 
addition, using the presence of EVR by week 4 as the criterion would allow better identification of the potential responders and recidivists, as well as permitting the duration of the treatment ( 24 or 48 weeks) to be individualized. Therefore, we can also do expand and improve treatment for individuals infected with genotype 1 .

All of these concepts are derived from studies of viral kinetics, although from a descriptive standpoint rather than from the use of mathematical calculations per se. This is desirable because it minimizes mathematical controversies and other limitations. However, it must be borne in mind that, even from this standpoint, an EVR is not a cure and that the kinetic model is still criticized for addressing only the plasma compartment [20]. As previously emphasized, EVR is directly linked to phase 1 of treatment, which lasts for 24 to 48 hours after the first dose of interferon is administered. Lower baseline viral loads, together with a rapid and vigorous response to the interferon stimulus, ensure an initial viremia reduction that will translate into a rapid and intense phase 1 . Consequently, in such patients, phase 2 begins at a viremia level that allows the elimination of all infected cells that produce new viral particles, thereby breaking the vicious cycle that perpetuates the HCV infection. For the slow responders, in whom viremia is reduced but still detectable, it is possible that prolonging the treatment provides better results and lower recurrence rates.

The rapidity at which events occur during phase 1 appears to be inconsistent with the pharmacokinetics described for pegylated interferon, whose time to absorption and peak plasma concentrations are preceded by the phenomena observed [21]. However, the viral kinetics observed, together with the interferon-induced gene activation [22], which immediately follows the treatment, underscores the crucial role that host status plays in obtaining an EVR. Furthermore, the results of an in vivo study conducted by Silva et al. [23] show that the two types of pegylated interferon (alpha 2a and alpha $2 b$ ) induce different responses. This might reflect differences in receptor binding related to the molecular weight of polyethylene glycol molecules (those with higher weights presenting less binding than those with lower weights) [24] or due to saturation of the IFNARs secundary to an excessive exposure. Silva et al. [23] found that patients treated with pegylated interferon alpha $2 \mathrm{~b}$ presenting greater expression of 2',5'-oligoadenylate synthetase and RNAdependent protein kinase, which reflected the intensity of $\mathrm{HCV}$ viremia reduction in the first week of treatment - a time point that was found to be crucial to determining treatment outcome. Therefore, further studies of interferon-induced immune responses and the pharmacokinetics of the drugs used in the treatment of chronic hepatitis $\mathrm{C}$, taking into consideration all aspects of parasite-host-drug interactions, should be encouraged. The findings of such studies could lead to the development of better and more comprehensive treatment strategies. Currently, HCV infections must be treated on a case-by-case basis (the so-called “individualization”), whether that leads to treatment strategies of shorter or longer duration, or even to early interruption of treatment, as has been proposed by Manns et al[25] to monoinfected patients and, by our group [15] to HIV coinfected patients. A schematic view is presented on Figure 4.

In conclusion, we recommend the use of EVR, and undetectable HCV RNA in particular, at week 4 of treatment. This criterion is an excellent predictor of SVR, as well as

Figure 4. Duration of therapy based on viral kinetics - fast or slow - and by liver disease and virus variables.

\section{Determinants of Virologic Response based on Viral Kinetics}

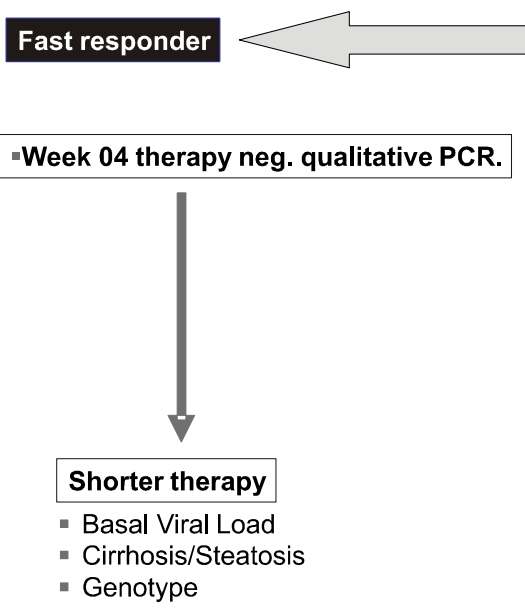

Slow responder

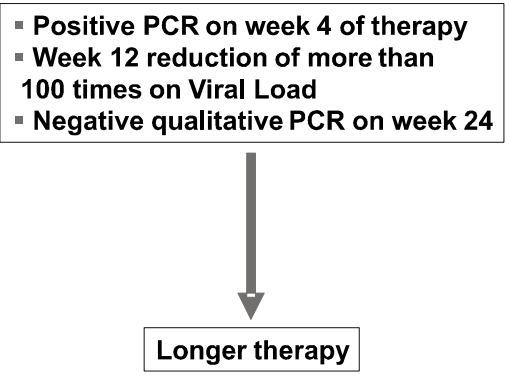

www.bjid.com.br 
offering the possibility of shortening the duration of treatment for individuals infected with genotypes 2 or 3 (12 weeks vs. 24 weeks), as well as for those infected with genotype 1 , having low viral loads and presenting EVR (24 weeks vs. 48 weeks), excluding patients with cirrhosis and severe steatosis who would require treatment of a standard, or even longer, duration.

\section{References}

1. Shepard C.W., Finelli L., Alter M.J. Global epidemiology of hepatitis C virus infection. Lancet Infect Dis 2005;5:558-67.

2. Araujo E.S.A., Silveira O.S. Hepatites na cidade de São Paulo in DST/Aids, a nova cara da luta contra a epidemia na cidade de São Paulo. 2003:55-68.

3. Nguyen T.T., Sedghi-Vaziri A., Wlkes L.B., et al. Fluctuations in viral load (HCV RNA) are relatively insignificant in untreated patients with chronic HCV infection. Journal of Viral Hepatitis 1996;3(2):75-8.

4. Pawlotsky J.M. Current and Future Concepts in Hepatitis C Therapy. Seminars in Liver Disease 2005;25:72-83.

5. Layden J.E., Layden T.J. How can Mathematics help us understand HCV? [Editorial]. Gastroenterology 2001;120(6):1546-9.

6. Tang K.H., Herrmann E., Cooksley H., et al. Relationship between early HCV kinetics and T-cell reactivity in chronic hepatitis $\mathrm{C}$ genotype 1 during peginterferon and ribavirin therapy. Journal of Hepatology 2006;43:776-82.

7. Ji X., Cheung R., Cooper S., et al. Interferon alfa regulated gene expression in patients initiating interferon treatment for chronic hepatitis C. Hepatology 2003;37:610-21.

8. Feld J.J., Hoofnagle J.H. Mechanism of action of interferon and ribavirin in treatment of hepatitis C. Nature 2005;436:967-72.

9. Neumann A.U., Lam N.P., Dahari H., et al. Hepatitis C viral dynamics in vivo and the antiviral efficacy of interferon- $\alpha$ therapy. Science 1998;282(5386):103-7.

10. Pham T.N.Q., Macparland S.A., Mulrooney P.M., et al. Hepatitis C Virus Persistence after spontaneous or treatmentinduced resolution of Hepatitis C. Journal of Virology 2004;78(11):5867-74.

11. Feld J.j., Liang T.J. HCV Persistence: cure is still a four letter word. Hepatology 2005;41:23-5.

12. Poynard T. Management protocols in chronic hepatitis $C$ in Hepatitis C and B Management and Treatment, Second Edition, Taylor and Frances Ed, 2005:19-34.
13. Ferenci P., Fried M.W., Shiffman M.L., et al. Predicting sustained virological responses in chronic hepatitis $C$ patients treated with peginterferon alfa-2a $(40 \mathrm{KD}) /$ ribavirin. Journal of Hepatology 2005;43:425-33.

14. Soriano V. Treatment of chronic hepatitis C in HIV-positive individuals: Selection of candidates. Journal of Hepatology 2006;44:S44-S8.

15. Araujo E.S.A., Neumann A.U., Courtouké C., et al. Early HCV kinetics predict sustained virological response to treatment with Peginterferon A2A and Ribavirin in Brazilian HIV/HCV co-infected patients. [P 38]. Abstracts book of the $12^{\text {th }}$ International Symposium on Hepatitis C and Related Viruses; 2005 Oct 2-6; Montreal, Canada. p.94.

16. Mangia A., Santoro R., Minerva N., et al Peginterferon Alfa 2b and Ribavirin for 12 vs. 24 weeks in HCV genotype 2 or 3, The New England Journal of Medicine 2005;325:2609-17.

17. Zeuzem S., Buti M., Ferenci P., et al Efficacy of 24 weeks treatment with peginterferon alfa-2b plus ribavirin in patients with chronic hepatitis $C$ infected with genotype 1 and low pretreatment viremia, Journal of Hepatology 2006;44:97-103.

18. Dalgard O., Bjoro K., Hellum K.B., et al. Treatment with pegylated interferon and ribavirin in HCV infection with genotype 2 or 3 for 14 weeks: a pilot study. Hepatology 2004;40:1260-5.

19. Craxi A., Cammà C. Treating patients with HCV genotype 1 and low viraemia: More than meets the eye. Journal of Hepatology 2006;44:4-7.

20. Lutchman G., Hoofnagle J.H. Viral kinetics in hepatitis C. Hepatology 2003;37(6):1257-9.

21. Foster G.R. Pegylated interferons: chemical and clinical differences. Alimentary Pharmacology and Therapeutics 2004;20(8):825-30.

22. Taylor M., Schaley J., Edenberg H., et al. Distinct differences in gene response between patients resistant to Interferon/Ribavirin therapy and those showing early vírus clearance [Oral Presentation 1]. Abstract book of the $11^{\text {th }}$ International Symposium on Hepatitis C Vírus and Related Viruses; 2004; Heidelberg, Germany. p.5.

23. Silva M., Poo J., Wagner F., et al. A randomized trial to compare the pharmacokinetic, pharmacodynamic, and antiviral effects of peginterferon alfa-2b and peginterferon alfa-2a in patients with chronic hepatitis C (COMPARE). Journal of Hepatology 2006; 45:204-13.

24. Dhalluin C., Ross A., Huber W., et al. Structural, Kinetic, and Thermodynamic analysis of the binding of the 40 Kda Peg-Interferon$\alpha 2 \mathrm{a}$ and its individual positional isomers to the extracellular domain of the receptor IFNAR2. Bioconjugate Chem 2005;16:518-27.

25. Manns M., Wedemeyer H., Cornberg M. Treating viral hepatitis C: efficacy, side effects, and complications. GUT 2006;55:1350-9. 